\title{
Potencial acuícola en áreas preandinas de la Región de La Araucanía: conflictos de uso con la actividad turística ${ }^{1}$
}

\author{
Denis Alfaro Castillo ${ }^{2 \dagger}$ y Fernando Peña-Cortés ${ }^{3}$
}

\begin{abstract}
RESUMEN
Se identificaron las áreas de uso y conflicto entre la salmonicultura y el turismo en el área preandina de la Región de La Araucanía en Chile. Para ello se determinaron variables de aptitud y de restricciones técnicas y legales que se integraron a través de un Sistema de Información Geográfica. Posteriormente, se evaluó la percepción de actores comunales frente a los dos tipos de actividades. Los resultados permitieron identificar tres sectores de conflicto espacial, todos en la comuna de Pucón. Se cuantificaron 245.113 ha potenciales de aptitud para la instalación de centros de cultivos de salmonídeos. Sin embargo, el estudio de percepción mostró que un amplio sector es renuente a la instalación de nuevos centros de cultivo. Por su parte, el turismo se percibe como una actividad que genera mayor empleabilidad en el sector, pero no es claro para la población los efectos ambientales de ambas actividades.
\end{abstract}

Palabras clave: Planificación territorial, conflicto de uso, acuicultura, turismo, percepción.

\begin{abstract}
Areas of use and conflict between salmon farming and tourism were identified in the preAndean area of the Araucanía Region, Chile. To do this, variables of suitability and of technical and legal restrictions were determined and incorporated using a Geographical Information System. Subsequently, the perception of actors in the communities in response to the two types of activity were evaluated. The results allowed three sectors of spatial conflict to be identified, all in the district of Pucón. A register was made of 245,113 ha which are potentially suitable for the installation of salmonid species cultivation centres. However the perception study revealed that a large sector of the population is against the installation of new cultivation centres. Meanwhile tourism is perceived as an activity which generates more local employment; however the environmental effects of the two types of activity are not clear to the population.
\end{abstract}

Key words: Territorial planning, conflict of use, aquaculture, tourism, perception.

1 Artículo financiado por la Dirección General de Investigación y Postgrado de la Universidad Católica de Temuco, Proyecto DGIPUCT N CD2010-01. Se agradece al Sr. Jimmy Pincheira por su valioso apoyo en la edición y revisión del trabajo; al Sr. Eduardo Fernández por la elaboración cartográfica y al Sr. Gonzalo Rebolledo por sus aportes metodológicos. Artículo recibido el 26 de enero de 2011, aceptado el 14 de enero de 2012 y corregido el 14 de marzo de 2012.
2 Laboratorio de Planificación Territorial, Facultad de Recursos Naturales, Universidad Católica de Temuco (Chile). La autora Denis Alfaro falleció durante el periodo de edición de este trabajo. El artículo es un reflejo de su especial interés y preocupación por contribuir, efectivamente, al logro de la sustentabilidad en los territorios de la Región de La Araucanía.

3 Laboratorio de Planificación Territorial, Núcleo de Investigación en Estudios Ambientales, Facultad de Recursos Naturales, Universidad Católica de Temuco (Chile). E-mail: fpena@uctemuco.cl 
El sector acuícola chileno basa su producción en el cultivo de salmones con valores de producción sobre las 650 mil toneladas, exportaciones por MUS\$2.241 en 2007 y se proyecta aumentar su producción actual a niveles cercanos a las 1.300 toneladas para el 2020 y 2.400 toneladas para el 2030 (Brugère y Ridler, 2005; Quiroz y Consultores Asociados, 2006; FAO, 2009). Sin embargo, para lograr las metas de producción propuestas, se hace necesario que la industria incremente la producción de ovas, alevines o smolts, etapas que necesariamente deben realizarse en un ambiente dulceacuícola, ejerciendo de esta manera una fuerte presión de uso sobre territorios que presentan pendientes suaves, con accesibilidad y proximidad a cursos fluviales superficiales permanentes de mediano torrente (esteros, riachuelos y quebradas) cuya cantidad y calidad son relevantes al momento de la localización de esta actividad (Pichara et al., 2007; Atland \& Bjerknes, 2009). Un espacio en el cual se han encontrado dichas condiciones, es el territorio preandino de La Araucanía, evidenciado en los últimos años por el fuerte incremento en el número de centros de cultivos regionales inscritos en el Registro Nacional de Acuicultura (38 centros en 1999; 88 centros año 2008) de los cuales, según los volúmenes de producción actuales, 35 corresponden a categoría industrial (SERNAPESCA, 2009), abasteciendo el 60\% de las ovas, alevines y smolts que demanda la actividad a nivel nacional (Pichara et al., 2007).

En este mismo espacio, se concentra el $50 \%$ de los 664 atractivos turísticos naturales existentes en la Región de La Araucanía; los que ofrecen variedad de atractivos y cuya actividad es intensa durante todo el año (LPTUCT, 2002). Sus atractivos naturales y culturales, de jerarquía internacional, explican la recepción de flujos turísticos crecientes provenientes tanto del país como del exterior (SERNATUR, 2009). Durante el año 2008, Chile generó un total de US\$2.030,5 millones como ingresos de divisas por turismo receptivo los que representan el $87,4 \%$ de los ingresos generados por las exportaciones de salmón y trucha. En el caso específico de la región de La Araucanía, las visitas recibidas a los atractivos naturales del Sistema Nacional de Áreas Protegidas del Estado (SNASPE), ubican a la región en el segundo lugar con respecto a turismo receptivo nacional $(12,5 \%)$ $y$, en cuarto lugar respecto al turismo receptivo extranjero $(4,7 \%)$. Las proyecciones del sector turístico mundial señalan que esta actividad crecerá a un ritmo del $4 \%$ anual (OMT, 2007) durante los próximos 10 años. En el caso de Chile, la World Travel and Tourism Council (WTTC, 2010), pronostica un notable desarrollo del turismo a un ritmo de $12,3 \%$ en los próximos 10 años lo cual permitirá que para el periodo 2008-2017 Chile ocupe el quinto lugar entre los 10 países de mayor crecimiento de la industria turística con US\$ MN 11.213,9 y el décimo entre los generadores de empleos con 1,3 millones para igual periodo. Al igual que la mayor parte de las industrias tradicionales, la acuicultura está en conflicto de uso creciente (Borja, 2002) con las actividades de agricultura, minería, servicios portuarios, navegación, pesca, esparcimiento, desarrollo industrial, vida salvaje y otros usos (Noakes et al., 2003; Jayanthi et al., 2006; Israel, 2007; Molinet et al., 2008).

Numerosos estudios describen conflictos entre la acuicultura y actividades productivas coexistentes en ambientes marinos (Union of British Columbian Indian Chiefs, 2004; Gowing et al., 2006a, 2006b; Molinet et al., 2008) y lacustres de agua dulce (Jayanthi et al., 2006; Israel, 2007). A nivel mundial, se han experimentado problemas sociales en países productores de salmón, como Chile, donde este cultivo ha creado trastornos sociales y cierta marginación de las personas con menores recursos, lo cual ha provocado una oposición a la acuicultura e incluso la destrucción deliberada de jaulas (Barrett et al., 2002; FAO, 2009). Por ejemplo, en Canadá, se ha extendido la opinión de que los cultivadores de salmón son una amenaza para los derechos de pesca de los aborígenes y los grupos de poblaciones indígenas han presionado al gobierno canadiense para que se oponga al desarrollo de toda nueva piscifactoría y mantenga su moratoria sobre el cultivo en jaulas de redes en el océano (Georgia Strait Alliance, 2002; Union of B.C. Indian Chiefs, 2004). En Chile, empresarios turísticos de varias regiones del país señalan que el turismo está enfrentando una realidad confrontacional con la acuicultura (OECD/ CEPAL, 2005). Históricamente, La Araucanía ha tenido un fuerte desarrollo turístico en toda el área andina y, más recientemente, un 
crecimiento acelerado de la salmonicultura. Esta relación ha generado conflictos locales ambientales y sociales ya que existe la percepción de que la acuicultura desarrollada en esta área estaría afectando la calidad de los atractivos turísticos, específicamente la calidad de las aguas que utiliza y del paisaje donde se emplaza y que, en síntesis, la ordenación del territorio está desvinculada de la problemática real y de las aspiraciones sociales existentes (IUCN, 2009).

El creciente y necesario desarrollo de la salmonicultura y del turismo en la Región de La Araucanía, los quiebres y conflictos que ambas actividades manifiestan originados por el uso de un mismo territorio, la ausencia de estudios tendientes a la evaluación del potencial de cultivo de salmonídeos en la región y los escasos trabajos de investigación que analizan la relación entre acuicultura y turismo en Chile, plantean la necesidad de analizar esta interacción. En este estudio, se identifican los espacios territoriales en conflicto actual, la aptitud y proyección espacial óptima del territorio para la salmonicultura en áreas preandinas de La Araucanía. Además, se analiza la percepción de actores territoriales sobre la compatibilidad, los aspectos legales y ambientales entre ambas actividades.

\section{Materiales y métodos}

\section{Descripción del área de estudio}

El área en estudio (Figura $N^{\circ} 1$ ) está conformada por las comunas de Lonquimay, Curacautín, Vilcún, Melipeuco, Villarrica, Pucón, Curarrehue y Cunco con una superficie de 1.363.395,7 ha. Estas comunas definidas geomorfológicamente como perteneciente a la Unidad Territorial Andina Volcánica de Alta Naturalidad y Aptitud Diversa (LPT-UCT, 2005), se caracterizan por presentar relieves abruptos pertenecientes a la cordillera de los Andes, numerosos hitos geográficos de origen glacial, volcanes, ríos, saltos de agua, alta ruralidad con aislamiento en los meses de invierno, bajo grado de intervención humana y alto grado de naturalidad.

En el área predominan los usos de conservación, turismo, ganadería, acuicultura y en menor medida forestal. El paisaje, producto de la combinación de lagos, ríos, volcanes con nieves eternas, y la presencia de bosques nativos, le han otorgado un alto valor territorial (LPT-UCT, 2002). El recurso agua, formado tanto por lagos de agua dulce (Villarrica, Caburgua, Colico, Huilipilún) y lagunas (Icalma, Galletué), está abastecido por ríos de aguas puras y oxigenadas provenientes de glaciares y volcanes nevados, con temperaturas entre $8^{\circ}$ y $15^{\circ} \mathrm{C}$ (Armesto y Smith-Ramírez, 1994; DGA, 2004a y b). 
Figura $\mathrm{N}^{\circ} 1$

Ubicación área de estudio

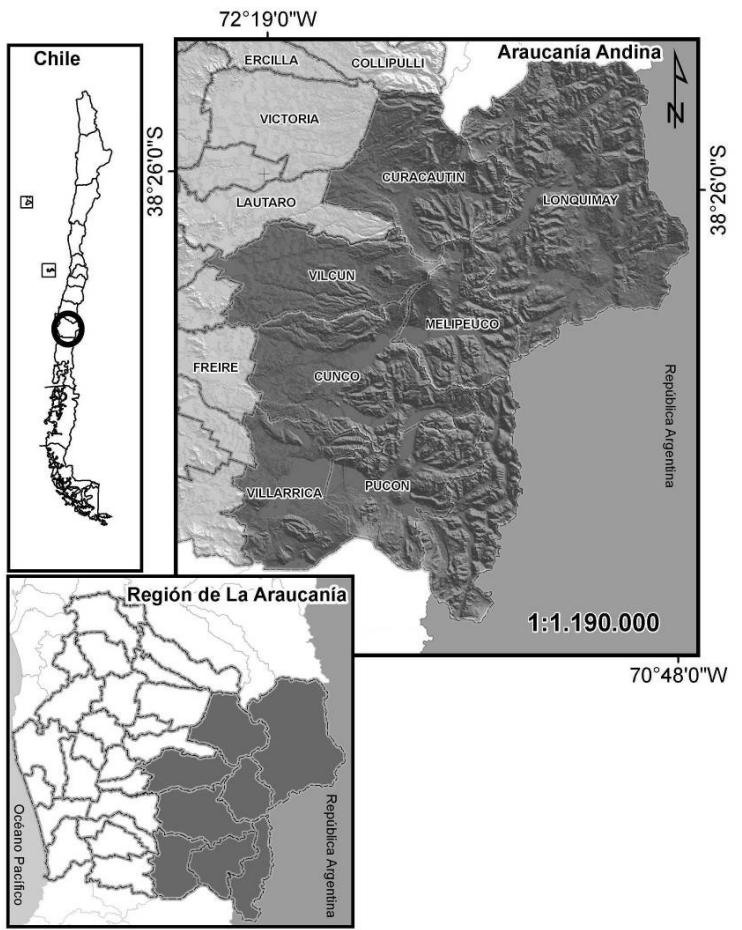

Fuente: Elaboración propia.

Identificación de las áreas de conflicto territorial actual

Se utilizó la base cartográfica del Instituto Geográfico Militar de Chile (IGM) escala 1:50.000 con un modelo de elevación digital de la misión SRTM (Shuttle Radar Topography Mission) de resolución de 60 m y cartografía vectorial base proporcionada por el Laboratorio de Planificación Territorial (LPT) de la Universidad Católica de Temuco. La información cartográfica básica de referencia de los suelos se obtuvo del Centro de Información de los Recursos Naturales y de la Corporación de Fomento de la Producción (CIREN-CORFO); las coordenadas geográficas de los centros de cultivo y de las áreas de atractivos turísticos se obtuvieron del Servicio Nacional de Pesca (SERNAPESCA) y Servicio Nacional de Turismo (SERNATUR), respectivamente. Para determinar las áreas de conflicto, se establecieron rangos de protección de las actividades turísticas de 500, 1.000, 2.000 y 3.000 m establecidos por SIGTUR (2002) para los atractivos turísticos de jerarquía local, regional, nacional e internacional, respectivamente; estos últimos según la clasificación de la Organización Mundial de Turismo; y en el caso de los centros de cultivo se consideró un área de protección de $250 \mathrm{~m}$. Esta información se procesó mediante la conversión a formato raster, y su posterior confección en capas temáticas. Se consideró la existencia de conflicto actual cuando las áreas de protección de ambas actividades se sobreponían entre sí.

Proyección espacial de las áreas óptimas para salmonicultura

Mediante un panel de expertos constituido por 18 personas (siete jefes de centros de cultivo, tres directores de servicios pertinentes, cinco académicos nacionales y tres académicos internacionales) se determinó la aptitud física y las restricciones del territorio 
para la salmonicultura así como la sobreposición de las actividades turísticas. La primera etapa se inició con la identificación de cinco variables de aptitud física del territorio para las actividades de salmonicultura: tipos de cursos de agua, accesibilidad (distancia a los caminos y vías de acceso), proximidad a cuerpos de agua superficiales permanentes, proximidad a centros urbanos y pendiente del terreno (Cuadro $N^{\circ} 1$ ). El panel también asignó ponderaciones mediante juicios de valor según la importancia asignada por ellos a cada variable (Geneletti, 2007, 2010). El tipo de curso de agua se consideró como la variable de mayor ponderación $(0,5)$ y la pendiente del terreno con la más baja $(0,03)$ (Cuadro $N^{\circ} 1$ ). Posteriormente, mediante modelo matricial y evaluación multicriterio (MCE) propuesto por Barredo (1996) y aplicado en Chile por Peña-Cortés y Mardones (1999) y Rivera (2001), las variables fueron integradas en el módulo AHP 11 (Analytical Hierarchy Process) de ArcGis para la construcción de una matriz de comparación por pares (Cuadro $N^{\circ} 1$ ), que permitió la diferenciación de los pesos de una variable en relación a otra, minimizando la carga de subjetividad de los criterios de evaluación iniciales (Geneletti, 2010).

La identificación de los cursos de agua que mantienen un caudal adecuado para el desarrollo de la salmonicultura a nivel industrial, se realizó mediante el programa ArcGis 9.2® y las extensiones ArcHydro, Patch Analyst y Spatial Analyst las cuales permitieron la delimitación de las cuencas, microcuencas y el ajuste de la red hidrográfica. La aptitud de cada cauce se definió por la relación entre la pendiente media (alta, media o baja) (Strahler, 1964; Mardones et al., 2007) y el orden de cada cauce definidos por
Strahler (1957) con valores de 1 a 8 (Cuadro $\left.N^{\circ} 1\right)$. Se estableció con aptitud muy alta, alta y media los cauces de orden 2, 3, 4 y 5 que corresponden a esteros y arroyos permanentes de características ritrónicas mientras que los cauces con valores de orden 1 e iguales o superiores a 6, 7 y 8, se definieron con aptitud media, baja o muy baja para la salmonicultura por corresponder a cursos de agua no permanentes o de características potamónicas, con alta carga de sedimentos en suspensión. Las variables de accesibilidad (distancia a los caminos y vías de acceso), proximidad a cuerpos de agua superficiales permanentes y a centros urbanos fueron definidas en base a rangos de distancia a caminos (asfaltados o de ripio), cuerpos de agua (superficiales y permanentes) y centros urbanos, respectivamente (Cuadro $\mathrm{N}^{\circ} 1$ ). La determinación de los rangos de aptitud según la pendiente del terreno se realizó según lo establecido por IUCN (2007, 2009) y Zuñiga et al. (2006), para la selección de sitio de centros de cultivo de peces.

Estas variables se agruparon en capas, mediante una suma lineal ponderada que permitió obtener la capa de aptitud física del territorio para la salmonicultura, estableciendo una escala numérica que incluye las variables y rangos de valor asociadas: Muy alta (5), Alta (4), Media (3), Baja (2) y Muy baja (1). El presente estudio no considera la calidad de agua como criterio para la selección de los cuerpos fluviales, dado que las características físicas y químicas de estos, se encuentran dentro de los valores óptimos requeridos para la salmonicultura (DGA, 2004a y b; Pichara et al., 2007; Atland \& Bjerknes, 2009). 
Cuadro $\mathrm{N}^{\circ} 1$

Variables de aptitud del territorio para la salmonicultura

\begin{tabular}{|c|c|c|c|c|c|c|c|}
\hline \multirow{2}{*}{\multicolumn{2}{|c|}{ Criterios }} & \multirow{2}{*}{ Peso } & \multicolumn{5}{|c|}{ Aptitud física para la salmonicultura } \\
\hline & & & Muy alta (5) & Alta (4) & Media (3) & Baja (2) & Muy baja (1) \\
\hline \multirow{3}{*}{$\begin{array}{l}\text { Tipos de } \\
\text { cursos de } \\
\text { agua }\end{array}$} & $\begin{array}{l}\text { Alta } \\
\text { pendiente } \\
\text { media }\end{array}$ & \multirow{3}{*}{0,50} & $\begin{array}{l}\text { Orden 2, } \\
3,4\end{array}$ & Orden 5,6 & Orden 7 & Orden 1, 8 & Orden $>8$ \\
\hline & $\begin{array}{l}\text { Media } \\
\text { pendiente } \\
\text { media }\end{array}$ & & Orden 3,4 & Orden 2, 5 & Orden 6 & Orden 1, 7 & Orden $>7$ \\
\hline & $\begin{array}{l}\text { Baja } \\
\text { pendiente } \\
\text { media }\end{array}$ & & Orden 3 & Orden 4 & $\begin{array}{l}\text { Ordenes } \\
2,5\end{array}$ & Orden 1, 6 & Orden $>6$ \\
\hline \multicolumn{2}{|c|}{$\begin{array}{l}\text { Accesibilidad (distancia } \\
\text { a los caminos y vías de } \\
\text { acceso) }\end{array}$} & 0,26 & $\begin{array}{l}0 \text { a } 2.000 \mathrm{~m} \\
\text { de caminos } \\
\text { asfaltados } \\
\text { y de } 0 \text { a } \\
500 \mathrm{~m} \text { de } \\
\text { un camino } \\
\text { secundario } \\
\text { s/pavimentar }\end{array}$ & $\begin{array}{l}2.000 \mathrm{a} \\
5.000 \mathrm{~m} \\
\text { de caminos } \\
\text { asfaltados } \\
\text { y de } 0 \mathrm{a} \\
500 \mathrm{~m} \\
\text { secundarios/ } \\
\text { sin } \\
\text { pavimentar }\end{array}$ & $\begin{array}{l}5.000 \text { a } \\
10.000 \mathrm{~m} \\
\text { de caminos } \\
\text { asfaltados } \\
\text { y de } 500 \\
\text { a } 1.000 \mathrm{~m} \\
\text { de camino } \\
\text { secundarios/ } \\
\text { sin } \\
\text { pavimentar }\end{array}$ & $\begin{array}{l}10.000 \mathrm{a} \\
20.000 \mathrm{~m} \\
\text { de camino } \\
\text { asfaltados } \\
\text { y de } 1.000 \\
\text { a } 2.000 \mathrm{~m} \\
\text { de caminos } \\
\text { secundarios/ } \\
\text { sin } \\
\text { pavimentar }\end{array}$ & $\begin{array}{l}>20.000 \mathrm{~m} \\
\text { de camino } \\
\text { asfaltados y } \\
>2.000 \mathrm{~m} \\
\text { de caminos } \\
\text { secundarios/ } \\
\text { sin } \\
\text { pavimentar }\end{array}$ \\
\hline \multicolumn{2}{|c|}{$\begin{array}{l}\text { Proximidad a cuerpos } \\
\text { de agua superficiales } \\
\text { permanentes }\end{array}$} & 0,13 & $<100 \mathrm{~m}$ & $100-200 m$ & $200-500 m$ & 500 a $1.000 \mathrm{~m}$ & $>1.000 \mathrm{~m}$ \\
\hline \multicolumn{2}{|c|}{$\begin{array}{l}\text { Proximidad a centros } \\
\text { urbanos }\end{array}$} & 0,08 & $5-10 \mathrm{~km}$ & $2-5 \mathrm{~km}$ & $10-20 \mathrm{~km}$ & $20-30 \mathrm{~km}$ & $>30 \mathrm{~km}$ \\
\hline \multicolumn{2}{|c|}{ Pendiente del terreno } & 0,03 & $<2 \%$ & 2 a $5 \%$ & 5 a $20 \%$ & 20 a $40 \%$ & $>40 \%$ \\
\hline
\end{tabular}

Fuente: Elaboración propia.

En la segunda etapa se determinaron las restricciones para el establecimiento de la salmonicultura mediante la generación de mapas binarios con las distintas variables técnicas, normativas y administrativas consideradas restrictivas, otorgando el valor 0 para excluir ese componente del análisis y el valor 1 cuando es permitido (Cuadro $\mathrm{N}^{\circ} 2$ ).
La aplicación cartográfica de las restricciones se realizó a través de un proceso de álgebra de mapas, en donde cada restricción o capa $(1 / 0)$ es multiplicada con la cobertura que contiene los valores de aptitud. 
Cuadro $\mathrm{N}^{\circ} 2$

Criterios de restricción del territorio para las actividades de salmonicultura

\begin{tabular}{|c|c|c|c|}
\hline $\begin{array}{l}\text { Criterios de } \\
\text { restricción }\end{array}$ & $\begin{array}{l}\text { Valor de } \\
\text { exclusión }(0)\end{array}$ & $\begin{array}{l}\text { Valor de } \\
\text { aptitud (1) }\end{array}$ & Observaciones \\
\hline $\begin{array}{l}\text { Áreas protegidas } \\
\text { (SNASPES) }\end{array}$ & Presencia & Ausencia & $\begin{array}{l}\text { Resolución } 0244-31.07 .80 \text { del } \\
\text { Ministerio de Salud establece distancia } \\
\text { mínima a SNASPES. }\end{array}$ \\
\hline $\begin{array}{l}\text { Cuerpos de agua } \\
\text { (lagos, lagunas) }\end{array}$ & Presencia & Ausencia & $\begin{array}{l}\text { DS N } N^{\circ} 371 / 01 \text { del Ministerio de } \\
\text { Defensa Nacional. }\end{array}$ \\
\hline Pendiente & $>20 \%$ & $<20 \%$ & $\begin{array}{l}\text { Variable de riesgo distancia a ladera } \\
\text { de alta pendiente }\end{array}$ \\
\hline $\begin{array}{l}\text { Proximidad a centros } \\
\text { urbanos }\end{array}$ & $<600 \mathrm{~m}$ & $>600 \mathrm{~m}$ & $\begin{array}{l}\text { Evaluado a } 600 \text { m de acuerdo a D.S. } \\
189-18.08 .05 \text { D.S. 05.01.08 RES } \\
2444-31.07 .80 .\end{array}$ \\
\hline Peligrosidad natural & Presencia & Ausencia & $\begin{array}{l}\text { LPT-UCT (2005), establece áreas de } \\
\text { riesgo natural. }\end{array}$ \\
\hline Tenencia de terreno & Mapuche & No mapuche & $\begin{array}{l}\text { Se utilizaron coberturas de mercedes } \\
\text { de propiedad y comunidades } \\
\text { mapuches actualizados, entregada por } \\
\text { CONADI. }\end{array}$ \\
\hline
\end{tabular}

Fuente: Elaboración propia.

En la tercera etapa, se elaboró el modelo espacial de aptitud óptima para la salmonicultura. Para ello se sobrepusieron las coberturas del modelo de aptitud física y las coberturas del modelo para desarrollo del turismo, elaboradas en el marco del estudio SIGTUR (2002). Este último, contiene coberturas con cuatro categorías de aptitud: nula, baja, media y alta. En tanto, las de aptitud para la salmonicultura (propuesta en este estudio) contiene cinco categorías, nula, baja, media, alta y muy alta. Atendiendo a estas diferencias, las capas de información se estandarizaron y transformaron a formato raster $(50 \times 50 \mathrm{~m})$, se reclasificaron y se efectuó una multiplicación de las capas con la herramienta Raster Calculator de la extensión Spatial Analyst de ArcGis 9.3.1. El resultado se reclasificó en cinco categorías, utilizando para ello el método de corte de histograma de Natural Breaks. De este modo, los niveles de conflicto entre el potencial acuícola y el potencial de desarrollo turístico se clasificó en cinco categorías: nulo, mínimo, medio, alto, máximo.

\section{Percepción del conflicto de compatibilidad de uso entre salmonicultura y turismo}

Con el objeto de determinar la percepción de actores relevantes de la comunidad, que representen a todos los segmentos de la población en las ocho comunas en estudio, se diseñó una encuesta semiestructurada la cual fue validada por un experto previo a su aplicación. La encuesta consistió en 19 preguntas cuyo diseño se centró en tres ámbitos de percepción: un primer ámbito, el conflicto, permite evaluar el rechazo o aprobación a la existencia de actividades productivas y la aptitud y compatibilidad percibida para el turismo y la salmonicultura. El segundo, el ambiental, considera el conocimiento de la normativa ambiental aplicable y el nivel de impacto que a juicio de los actores consultados, se atribuyen a las actividades productivas en estudio. El tercer y último, la empleabilidad, evidencia la percepción de los sectores encuestados sobre el nivel de em- 
pleo con que las actividades de salmonicultura y turismo contribuyen en cada comuna. La consulta se aplicó a cuatro representantes del gobierno comunal (Alcalde; Director de la Secretaría Comunal de Planificación [SECPLAC]; Director de la Unidad de Desarrollo Local [UDEL]; y Director de la Dirección de Desarrollo Comunal, [DIDECO]), dos representantes del sector privado (Presidente de la Cámara de Comercio y Presidente de Cámara de Turismo) y dos representantes de la comunidad (Presidente de la Unión Comunal de Junta de Vecinos Rurales y Presidente de la Unión Comunal de Junta de Vecinos Urbanas), en total 64 encuestas.

\section{Resultados}

\section{Determinación de áreas de conflicto territorial actual}

Las ocho comunas en estudio concentran el $65 \%$ de los centros de cultivos de salmonídeos y el $50 \%$ de los atractivos turísticos naturales en la región de la Araucanía (SERNAPESCA, 2009; SERNATUR, 2009; SUBPESCA, 2009). Las áreas con mayor número de centros de cultivo corresponden a la comuna de Villarrica, Pucón, Melipeuco, Curacautín, y Cunco y se ubican en torno a los tributarios del lago Villarrica y río Allipén
(Figura $\mathrm{N}^{\circ} 2$ ). Para sus procesos productivos captan agua proveniente de cuerpos fluviales menores como ríos, arroyos y esteros permanentes y descargan sus efluentes en los mismos. Respecto al turismo, las actividades turísticas se desarrollan en dos áreas existentes en el sector en estudio (SERNATUR, 2009), las cuales se caracterizan por poseer atractivos turísticos de jerarquía internacional de acuerdo a la clasificación de la Organización Mundial de Turismo (OMT). Estas son el "Área turística Araucanía Andina" y el "Área turística Villarrica-Pucón". La primera está conformada por las comunas de Victoria, Curacautín, Lonquimay, Vilcún, Cunco y Melipeuco mientras que la segunda comprende las comunas de Cunco, Pucón, Villarrica y Curarrehue (SERNATUR, 2007).

La superposición de las áreas donde se desarrollan actividades turísticas y de salmonicultura, revela la existencia de cuatro centros de cultivo en conflicto de uso con actividades turísticas, ambas ubicadas en la comuna de Pucón (Figura $N^{\circ}$ 2D). Sin embargo, resulta relevante que, salvo estos cuatro centros de cultivo, los centros restantes se encuentran en sectores donde no existe uso turístico. 
Figura $\mathrm{N}^{\circ} 2$

Áreas actuales de conflicto de uso entre las actividades turísticas y de salmonicultura en la área preandina de La Araucanía.
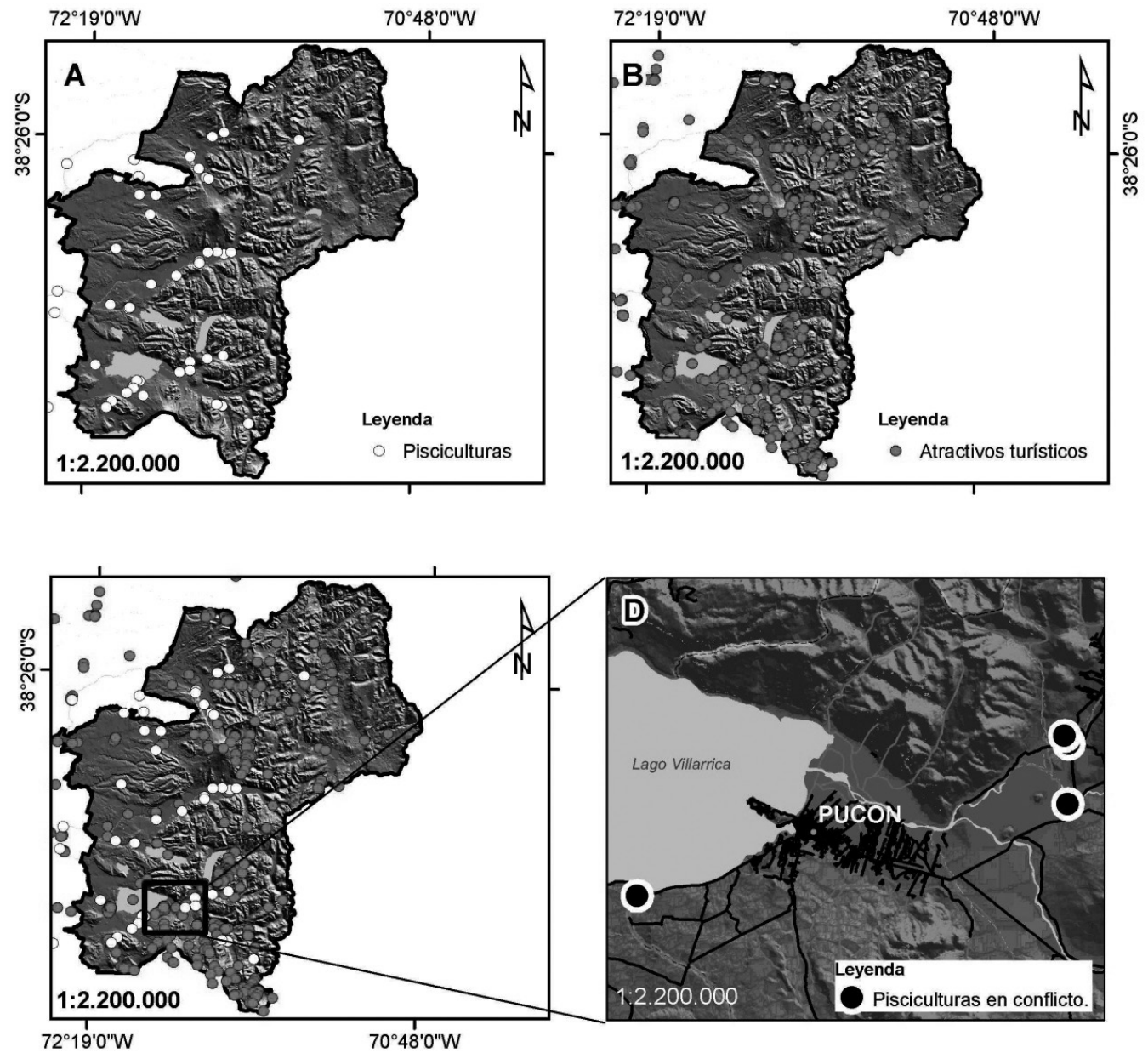

Fuente: Elaboración propia.

A: Centro de cultivos salmonídeos (pisciculuras).

B: Atractivos turísticos.

C: Área de traslape de los centros de cultivos salmonídeos con atractivos turísticos.

D: Áreas de conflicto de uso entre las actividades turísticas y de salmonicultura.

Proyección espacial de las áreas óptimas para salmonicultura

El modelo de aptitud física indica la existencia de 347.748 ha con aptitud alta y 17.708 ha con aptitud muy alta para la salmonicultura en el área preandina de la Región de La Araucanía. Esto arroja una superficie conjunta de 365.456 ha (Cuadro $\mathrm{N}^{\circ}$
3, Figura $\left.\mathrm{N}^{\circ} 3 \mathrm{~A}\right)$. Luego, al incluir las restricciones técnicas y normativas que existen respecto a la actividad (Figura $N^{\circ} 3 \mathrm{~B}$ ), esta área se reduce a 245.108 ha como óptima para la salmonicultura (aptitud muy alta y alta), lo cual representa una reducción del 32,9\% respecto al área con aptitud física total. El desglose por comuna señala que aun cuando las comunas de Villarrica y Pucón concentran 
el mayor número de centros de cultivo existentes (10 y 8 centros respectivamente), las áreas con mayor aptitud (muy alta y alta) para el desarrollo de la salmonicultura, considerando las restricciones, se encuentran en las comunas de Lonquimay, Cunco y Curacautín.

Cuadro $\mathrm{N}^{\circ} 3$

Superficie con aptitud para la salmonicultura

\begin{tabular}{|c|c|c|c|c|c|c|c|c|c|c|}
\hline \multirow{2}{*}{$\begin{array}{l}\text { Nivel de } \\
\text { aptitud }\end{array}$} & & \multicolumn{9}{|c|}{ Superficie de aptitud para la salmonicultura (ha/comuna) } \\
\hline & & Cunco & Curacautín & Curarrehue & Lonquimay & Melipeuco & Pucón & Vilcún & Villarrica & TOTAL \\
\hline \multirow{2}{*}{ 5: Muy Alta } & $\mathrm{s} / \mathrm{r}$ & $2.953,3$ & $2.530,7$ & $1.184,5$ & $1.218,4$ & 540,6 & $2.776,4$ & $2.350,4$ & $4.153,5$ & 17.708 \\
\hline & $\mathrm{c} / \mathrm{r}$ & $2.164,3$ & $2.322,5$ & 896,3 & 965,9 & 363,3 & 754,8 & $2.067,8$ & $1.555,8$ & 11.091 \\
\hline \multirow{2}{*}{ 4: Alta } & $\mathrm{s} / \mathrm{r}$ & $51.182,6$ & $45.301,6$ & $34.227,3$ & $76.748,3$ & $33.563,2$ & $38.596,7$ & $34.605,6$ & $33.522,7$ & 347.748 \\
\hline & $\mathrm{c} / \mathrm{r}$ & $41.015,3$ & $34.339,2$ & $19.828,8$ & $49.995,7$ & $18.302,8$ & $21.457,8$ & $28.010,0$ & $21.067,8$ & 234.017 \\
\hline \multirow{2}{*}{ 3: Media } & $\mathrm{s} / \mathrm{r}$ & $78.348,1$ & $58.434,2$ & $33.487,2$ & $123.417,7$ & $31.991,3$ & $43.237,2$ & $73.473,3$ & $68.956,9$ & 511.346 \\
\hline & $\mathrm{c} / \mathrm{r}$ & $62.318,8$ & $45.871,1$ & $18.356,3$ & $81.947,3$ & $14.891,3$ & $22.320,1$ & $60.858,4$ & $43.781,8$ & 350.345 \\
\hline \multirow{2}{*}{ 2: Baja } & $\mathrm{s} / \mathrm{r}$ & $56.069,4$ & $58.032,5$ & $46.936,3$ & $192.195,7$ & $43.812,2$ & $39.826,4$ & $27.780,7$ & $21.113,8$ & 485.767 \\
\hline & $\mathrm{c} / \mathrm{r}$ & $44.812,1$ & $41.200,7$ & $18.654,4$ & $131.446,9$ & $14.167,2$ & $22.031,6$ & $21.511,6$ & $18.336,8$ & 312.161 \\
\hline \multirow{2}{*}{ 1: Muy baja } & $\mathrm{s} / \mathrm{r}$ & 15,3 & 221,7 & 88,3 & 171,6 & - & - & 196,6 & 45,9 & 739 \\
\hline & $\mathrm{c} / \mathrm{r}$ & $38.258,3$ & $40.787,3$ & $58.187,7$ & $129.396,0$ & $62.182,6$ & $57.872,4$ & $25.958,8$ & $43.050,6$ & 455.694 \\
\hline \multicolumn{2}{|l|}{ Total general } & $188.568,7$ & $164.520,7$ & $115.923,6$ & $393.751,7$ & $109.907,3$ & $124.436,8$ & $138.406,6$ & $127.792,8$ & 1.363 .308 \\
\hline \multicolumn{2}{|l|}{$\begin{array}{l}\mathrm{N}^{\circ} \text { centros } \\
\text { industriales }\end{array}$} & 6 & 7 & 4 & 1 & 7 & 8 & 4 & 10 & 47 \\
\hline
\end{tabular}

$\mathrm{s} / \mathrm{r}$ : aptitud física sin restricciones de uso

c/r: aptitud física con restricciones de uso

Fuente: Elaboración propia.

\section{Áreas de conflicto potencial para el} desarrollo de la salmonicultura

La superposición de los modelos de aptitud física, incluyendo las restricciones para la localización de centros de cultivos salmonídeos (áreas naturales protegidas, regulación de áreas aptas para acuicultura, aspectos morfogeográficos, proximidad a centros urbanos y sectores poblados, zonas de riesgos naturales y propiedad indígena) (Figura $\mathrm{N}^{\circ}$ $3 \mathrm{~A})$, muestra que las actividades turísticas se encuentran ubicadas justamente en áreas con restricción para la salmonicultura (Figura $\mathrm{N}^{\circ}$ 3B) y, que áreas con aptitud muy alta (5) y alta (4) para la salmonicultura se encuentran en zonas alejadas de las actuales actividades turísticas (Figura №3B). Finalmente, las áreas óptimas para el desarrollo de la salmonicultura sin conflicto con el desarrollo del turismo llega a 563.045 ha ( $41 \%$ de nulo conflicto), mientras que en las zonas de alta y máxima conflictividad es notablemente menor, con 32.421 ha $(2 \%)$ y 14.646 ha $(1 \%)$, respectivamente (Figura $\mathrm{N}^{\circ} 3 \mathrm{C}$ ). 
Figura $\mathrm{N}^{\circ} 3$

Modelo espacial de aptitud óptima para la salmonicultura
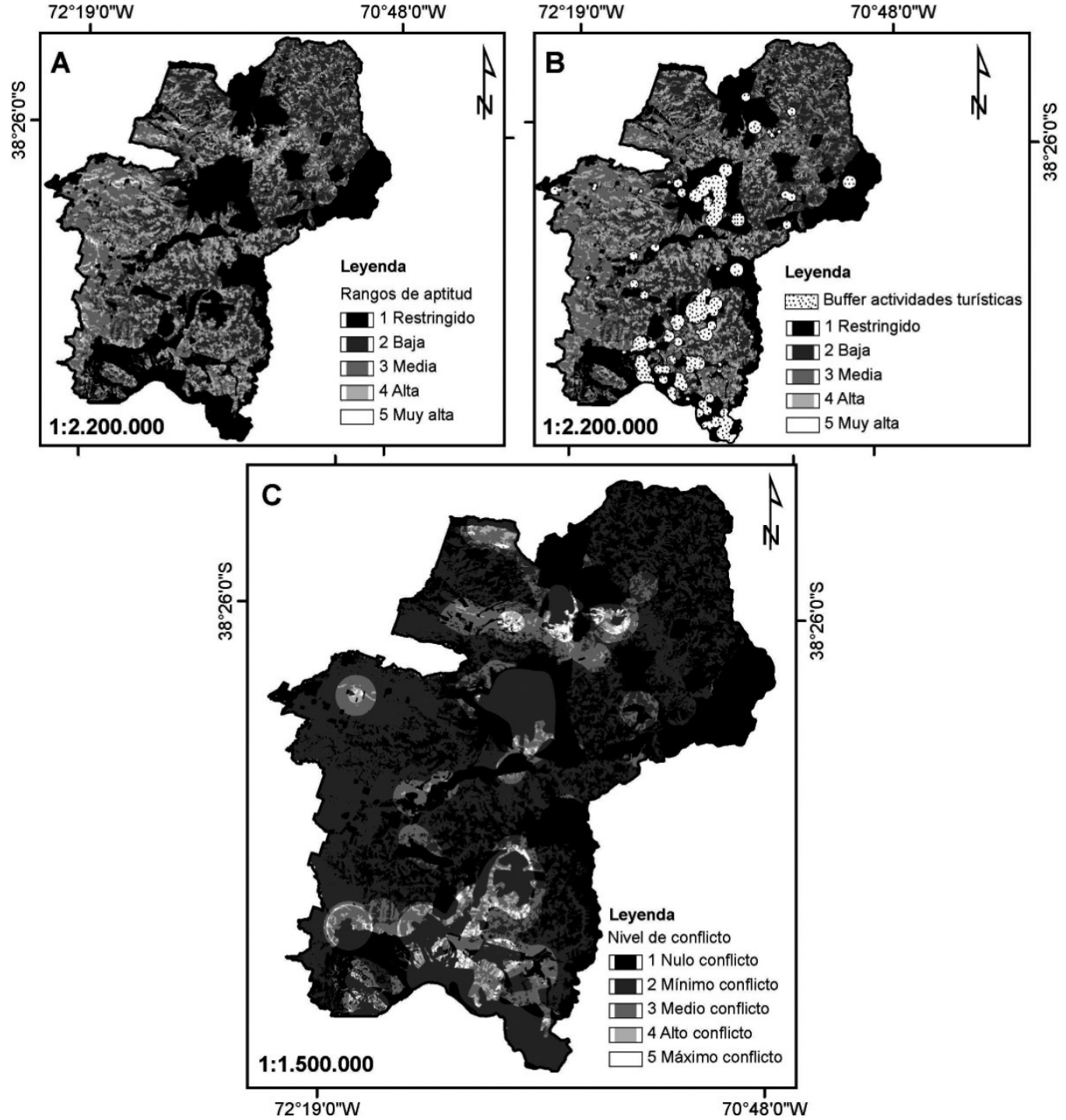

Fuente: Elaboración propia.

A: Aptitud física con restricciones para la localización potencial de Centros de cultivos salmonídeos.

B: Con áreas de influencia de las actividad turísticas actual.

C: Nivel de conflicto entre el potencial acuícola y el potencial de desarrollo turístico 
Percepción del conflicto de compatibilidad de uso

Los resultados de las encuestas aplicadas para evaluar el nivel de percepción de la población en cuanto a la compatibilidad del uso acuícola con el turístico, se obtuvieron sobre la base de un $68,7 \%$ de respuesta efectiva, sobre un total de 64 encuestas. De estas, 12 encuestas $(37,5 \%)$ no fueron respondidas por los representantes del Gobierno Comunal, lo mismo ocurrió con cinco encuestas del sector privado $(31,3 \%)$ y tres $(18,8 \%)$ de los representantes de la comunidad organizada.

Todos los sectores encuestados consideran que el territorio tiene mayor aptitud para el turismo que para la salmonicultura y al momento de elegir entre ambas actividades, prefieren a la primera. Ninguno de los sectores encuestados prefirió el desarrollo de la salmonicultura como única actividad y menos del 50\% (45,0\% Gobierno, 27,7\% privados y $15,4 \%$ Comunidad organizada) desea que se desarrollen ambas. Un análisis más detallado de esta información (Cuadro $N^{\circ} 4$ ) permite observar que la comunidad organizada es el grupo que tiene mayor conocimiento de las actividades turísticas y de los centros de cultivos existentes en su comuna $(100 \%$ y $84,6 \%$ respectivamente); este sector también considera que su territorio tiene aptitud física para ambas actividades $(77 \%$ salmonicultura; $100 \%$ Turismo), pero que estas no son compatibles entre sí $(61,6 \%)$ y, en general, prefieren que se desarrolle el turismo por sobre la salmonicultura $(84,6 \%)$.

Es interesante observar que, el grado de conocimiento por parte de los representantes del gobierno acerca de la presencia de de centros de cultivos y atractivos turísticas fue de $80 \%$ y $95 \%$, respectivamente. Por su parte, el sector privado presenta niveles de respuesta que variaron entre $81,8 \%$ y $100 \%$, respectivamente, mientras que para los representantes de la comunidad estos valores algo más alto, variando entre $100 \%$ y $84,6 \%$. Esto evidencia que la comunidad tiende a conocer más ampliamente su entrono local.

\section{Cuadro $\mathrm{N}^{\circ} 4$}

Percepción de la existencia de actividades, aptitud y compatibilidad del territorio para el turismo y la salmonicultura (en porcentajes)

\begin{tabular}{|c|c|c|c|c|c|c|c|c|c|c|}
\hline \multirow{2}{*}{\multicolumn{2}{|c|}{ Pregunta }} & \multicolumn{3}{|c|}{ Gobierno } & \multicolumn{3}{|c|}{ Privado } & \multicolumn{3}{|c|}{ C } \\
\hline & & \% sí & $\%$ no & $\% \mathrm{~ns}$ & \% sí & $\%$ no & $\% \mathrm{~ns}$ & \% sí & $\%$ no & $\% \mathrm{~ns}$ \\
\hline \multicolumn{2}{|c|}{$\begin{array}{l}\text { ¿Existen centros de cultivo de } \\
\text { peces en la comuna? }\end{array}$} & 80,0 & 10,1 & 9,9 & 81,8 & 9,2 & 9,0 & 84,6 & 15,4 & 0,0 \\
\hline \multicolumn{2}{|c|}{$\begin{array}{l}\text { ¿Existen en la comuna } \\
\text { atractivos turísticos? }\end{array}$} & 95,0 & 5,0 & 0,0 & 100,0 & 0,0 & 0,0 & 100,0 & 0,0 & 0,0 \\
\hline \multirow{2}{*}{\begin{tabular}{|l} 
Considera \\
Ud. que la \\
comuna tiene \\
condiciones \\
para el \\
desarrollo de:
\end{tabular}} & Salmonicultura & 88,2 & 8,2 & 3,7 & 91,0 & 0,0 & 9,0 & 77,0 & 15,4 & 7,6 \\
\hline & Turismo & 100,0 & 0,0 & 0,0 & 100,0 & 0,0 & 0,0 & 100,0 & 0,0 & 0,0 \\
\hline \multicolumn{2}{|c|}{$\begin{array}{l}\text { Considera que la salmonicultura } \\
\text { y el turismo son actividades } \\
\text { compatibles en la comuna? }\end{array}$} & 65,0 & 30,1 & 5,0 & 54,6 & 45,4 & 0,0 & 38,4 & 61,6 & 0,0 \\
\hline \multirow{3}{*}{$\begin{array}{l}\text { Prefiere que } \\
\text { en la comuna } \\
\text { se desarrolle: }\end{array}$} & Turismo & 55,0 & 22,5 & 0,0 & 72,8 & 13,6 & 0,0 & 84,6 & 7,7 & 0,0 \\
\hline & Salmonicultura & 0,0 & 50,0 & 0,0 & 0,0 & 50,0 & 0,0 & 0,0 & 50,0 & 0,0 \\
\hline & Ambas & 45,0 & 27,5 & 0,0 & 27,2 & 36,4 & 0,0 & 15,4 & 42,3 & 0,0 \\
\hline
\end{tabular}

ns: no sabe.

Fuente: Elaboración propia. 
La percepción de los aspectos ambientales de las actividades productivas (Cuadro $\mathrm{N}^{\circ}$ 5) evidencia bajo nivel de conocimiento de las normativas ambientales existentes y aplicables a las actividades turísticas (50,1\% Gobierno, 45,6\% Privado y $23 \%$ Comunidad) y de salmonicultura (45,1\% Gobierno, $18,2 \%$ privado y $7,6 \%$ Comunidad), particularmente en la comunidad y privados. Los resultados indican que no existe claridad en el ámbito ambiental afectado por estas actividades y consideran que, en términos generales, el turismo tiene un efecto menor que la salmo- nicultura sobre el medio ambiente. El 49,9\% de los representantes de Gobierno y el 63,8\% del sector privado consideran que los centros de cultivos impactan solo sobre la calidad del agua, mientras la percepción de la comunidad organizada es más diversa ya que el $53,9 \%$ considera que los centros de cultivos de salmones no solo impactan sobre el agua, sino también sobre el suelo y el aire.

\section{Cuadro $\mathrm{N}^{\circ} 5$}

Percepción medioambiental de las actividades turísticas y de salmonicultura (en porcentaje)

\begin{tabular}{|c|c|c|c|c|c|c|c|c|c|}
\hline \multirow[b]{2}{*}{ Preguntas } & \multicolumn{3}{|c|}{ Gobierno } & \multicolumn{3}{|c|}{ Privado } & \multicolumn{3}{|c|}{ Comunidad } \\
\hline & $\begin{array}{l}\% \\
\text { sí }\end{array}$ & $\begin{array}{l}\% \\
\text { no }\end{array}$ & $\begin{array}{l}\% \\
\mathrm{~ns}\end{array}$ & $\begin{array}{l}\% \\
\text { sí }\end{array}$ & $\begin{array}{l}\% \\
\text { no }\end{array}$ & $\begin{array}{l}\% \\
\mathrm{~ns}\end{array}$ & \% & $\begin{array}{l}\% \\
\text { no }\end{array}$ & $\begin{array}{l}\% \\
\mathrm{~ns}\end{array}$ \\
\hline $\begin{array}{l}\text { ¿En la comuna, se practica } \\
\text { el respeto al medio } \\
\text { ambiente? }\end{array}$ & 40,0 & 55,0 & 5,0 & 18,2 & 72,6 & 9,2 & 38,4 & 53,8 & 7,8 \\
\hline $\begin{array}{l}\text { ¿A su juicio, se aplica la } \\
\text { normativa ambiental en las } \\
\text { actividades turísticas? }\end{array}$ & 50,1 & 35,0 & 14,9 & 45,6 & 36,2 & 18,2 & 23,0 & 69,2 & 7,8 \\
\hline $\begin{array}{l}\text { ¿A su juicio, se aplica la } \\
\text { normativa ambiental en las } \\
\text { actividades de cultivo de } \\
\text { peces? }\end{array}$ & 45,0 & 45,0 & 10,1 & 18,2 & 72,6 & 9,2 & 7,6 & 84,6 & 7,8 \\
\hline $\begin{array}{l}\text { ¿El turismo impacta sobre } \\
\text { la calidad del agua? }\end{array}$ & 20,0 & 75,0 & 5,0 & 9,2 & 90,8 & 0,0 & 30,7 & 69,3 & 0,0 \\
\hline $\begin{array}{l}\text { ¿El turismo impacta sobre } \\
\text { la calidad del agua, suelo } \\
\text { y aire? }\end{array}$ & 29,9 & 65,1 & 5,0 & 9,2 & 90,8 & 0,0 & 7,6 & 92,4 & 0,0 \\
\hline $\begin{array}{l}\text { ¿La salmonicultura impacta } \\
\text { sobre la calidad del agua? }\end{array}$ & 49,9 & 45,1 & 5,0 & 63,8 & 36,2 & 0,0 & 15,4 & 84,6 & 0,0 \\
\hline $\begin{array}{l}\text { ¿La salmonicultura impacta } \\
\text { sobre la calidad del agua, } \\
\text { suelo y aire? }\end{array}$ & 9,9 & 85,1 & 5,0 & 27,4 & 72,6 & 0,0 & 53,9 & 46,1 & 0,0 \\
\hline
\end{tabular}

ns: no sabe.

Fuente: Elaboración propia.

En las respuestas obtenidas sobre el empleo generado por las actividades productivas (Cuadro $\mathrm{N}^{\circ} 6$ ), todos los sectores encuestados señalan que los centros de cultivo de peces son una fuente de empleo importante en las respectivas comunas $(89,9 \%$ Gobierno, $72,6 \%$ Privado y $84,5 \%$ Comunidad), valores que disminuyen cuando se formula la misma consulta sobre el turismo $(65,6 \%$ Gobierno, $63,6 \%$ privado y $53,9 \%$ Comunidad). Sin embargo, al consultar sobre el nivel de impacto que ocasiona cada una de estas actividades sobre la empleabilidad comunal, pocos representantes de los sectores encuestados 
reconocen en la salmonicultura un impacto alto sobre el empleo ( $0 \%$ Gobierno, 9, $1 \%$ Privado y $7,7 \%$ Comunidad), mientras que para el turismo el nivel de las respuestas frente a la misma consulta es bastante más alto por sector $(40,0 \%$ Gobierno, $23,1 \%$ Privado y $40,0 \%$ Comunidad).
En síntesis, los sectores encuestados reconocen mayoritariamente que el turismo genera una empleabilidad de impacto de nivel regular a alto mientras que la salmonicultura genera un impacto regular a bajo, siendo la comunidad organizada el sector que presenta un mayor nivel de respuestas.

Cuadro $\mathrm{N}^{\circ} 6$

Percepción de la empleabilidad generada por las actividades de turismo y salmonicultura (en porcentajes)

\begin{tabular}{|c|c|c|c|c|c|c|c|c|c|c|c|c|}
\hline \multirow[b]{2}{*}{ Preguntas } & \multicolumn{4}{|c|}{ GOBIERNO } & \multicolumn{4}{|c|}{ PRIVADO } & \multicolumn{4}{|c|}{ COMUNIDAD } \\
\hline & \multicolumn{2}{|c|}{$\begin{array}{l}\% \\
\text { sí }\end{array}$} & $\begin{array}{l}\% \\
\text { no }\end{array}$ & $\begin{array}{l}\% \\
\mathrm{~ns}\end{array}$ & $\begin{array}{l}\% \\
\text { sí }\end{array}$ & \multicolumn{2}{|c|}{$\begin{array}{l}\% \\
\text { no }\end{array}$} & $\begin{array}{l}\% \\
\mathrm{~ns}\end{array}$ & \multicolumn{2}{|c|}{$\%$} & $\begin{array}{l}\% \\
\text { no }\end{array}$ & $\begin{array}{l}\% \\
\mathrm{~ns}\end{array}$ \\
\hline $\begin{array}{l}\text { ¿Los centros de cultivo } \\
\text { generan empleo para la } \\
\text { comuna? }\end{array}$ & \multicolumn{2}{|c|}{89,9} & 10,1 & 0,0 & 72,6 & \multicolumn{2}{|c|}{18,2} & 9,2 & \multicolumn{2}{|c|}{84,5} & 7,7 & 7,7 \\
\hline $\begin{array}{l}\text { ¿Las actividad turística } \\
\text { genera empleo para la } \\
\text { comuna? }\end{array}$ & \multicolumn{2}{|c|}{65,0} & 25,0 & 10,1 & 63,6 & \multicolumn{2}{|c|}{36,4} & 0,0 & \multicolumn{2}{|c|}{53,9} & 46,1 & 0,0 \\
\hline \multirow[b]{2}{*}{ Preguntas } & \multicolumn{4}{|c|}{ GOBIERNO } & \multicolumn{4}{|c|}{ PRIVADO } & \multicolumn{4}{|c|}{ COMUNIDAD } \\
\hline & $\begin{array}{c}\% \\
\text { alto }\end{array}$ & $\begin{array}{c}\% \\
\text { regular }\end{array}$ & $\begin{array}{c}\% \\
\text { bajo }\end{array}$ & $\begin{array}{l}\% \\
\mathrm{~ns}\end{array}$ & $\begin{array}{c}\% \\
\text { alto }\end{array}$ & $\begin{array}{c}\% \\
\text { regular }\end{array}$ & $\begin{array}{c}\% \\
\text { bajo }\end{array}$ & $\begin{array}{l}\% \\
\mathrm{~ns}\end{array}$ & $\begin{array}{c}\% \\
\text { alto }\end{array}$ & $\begin{array}{c}\% \\
\text { regular }\end{array}$ & $\begin{array}{c}\% \\
\text { bajo }\end{array}$ & $\begin{array}{l}\% \\
\text { ns }\end{array}$ \\
\hline $\begin{array}{l}\text { La salmonicultura gene- } \\
\text { ra una empleabilidad de } \\
\text { impacto }\end{array}$ & 0,0 & 50,1 & 45,0 & 5,0 & 9,1 & 27,3 & 45,5 & 18,1 & 7,7 & 30,7 & 53,8 & 7,7 \\
\hline $\begin{array}{l}\text { El turismo genera una } \\
\text { empleabilidad de im- } \\
\text { pacto }\end{array}$ & 40,0 & 40,0 & 20,0 & 0,0 & 23,1 & 38,5 & 23,1 & 0,0 & 38,5 & 46,1 & 15,4 & 0,0 \\
\hline
\end{tabular}

ns: no sabe

Fuente: Elaboración propia.

\section{Discusión}

Como cualquier otra actividad económica, la acuicultura necesita del uso y transformación de recursos naturales (espacio, agua, comida, semillas, larvas, etc.) para generar un producto (peces, moluscos, crustáceos, etc.). El crecimiento de esta actividad depende de la expansión de las zonas de cultivo, el aumento de la densidad de individuos, el número de centros de cultivo y el uso de fuentes de alimento exógeno (Soto et al., 2008). Estos requerimientos del sector podrían llevar a impactos negativos sobre el medio ambiente y sobre sectores de la sociedad, en el caso de que este desarrollo fuese desordenado o mal administrado (Pérez et al., 2002; Suryanata y Unemoto, 2005; Jayanthi et al., 2006; Israel, 2007). También, puede ocurrir que la administración pública (o la sociedad civil) impida el desarrollo de una actividad viable desde el punto de vista técnico-económico y territorial pero que se considera nociva para otros intereses (Borja, 2002; Primavera, 2006). Más aún, cuando no existe planificación en torno al uso del territorio o cuando no es participativa, los usos quedan liberados frente al mercado y son la oferta y la demanda quienes ordenan los espacios según intereses de corto plazo. El resultado, es la realización 
de actividades y proyectos que muchas veces resultan negativos para el medio ambiente y el modo de vida local lo cual se refleja en problemas de sustentabilidad en el mediano y largo plazo (Baeriswyl, 2001; Peña-Cortés et al., 2009). En el caso específico de la relación acuicultura y turismo, se genera una competencia negativa dada por la percepción que ambas actividades usan un mismo espacio donde los residuos generados en los centros de cultivos pueden tener efectos negativos en los recursos naturales disminuyendo su calidad y cantidad, así como en el mantenimiento de la naturalidad del paisaje, de la vida salvaje y de la protección de la naturaleza que son elementos claves para el turismo (Pérez et al., 2005; Gowing et al., 2006a y b).

Según el Registro Nacional de Acuicultura del SERNAPESCA, durante los últimos 10 años en la Región de La Araucanía, el sector acuícola ha presentado un fuerte incremento en el número de Autorizaciones de Acuicultura otorgadas desde el año 1999 (38) al 2008 (88). Este aumento ocurre como consecuencia de la necesidad del mercado de abastecerse de ovas, alevines y smolt para cumplir con las metas de producción proyectadas al año 2020. Los centros que actualmente están en operación en el área en estudio, producen el $60 \%$ de las ovas, el $30 \%$ de los alevines y entre el 9 al $12 \%$ de los smolts que sustenta la salmonicultura del país (Pichara et al., 2007). Esta situación se ha traducido en la instalación de centros de cultivos en sectores donde coexisten actividades turísticas, con el consecuente surgimiento de conflictos entre ambas actividades ya que ambas comparten los mismos recursos naturales (Borja, 2002; Bryceson, 2002; Noakes et al., 2003; Buitrago et al., 2005; Macías et al., 2005; Radiarta et al., 2008; Halide et al., 2009 y Hossain et al., 2009). Sin embargo, la región presenta extensas áreas con aptitud física para la instalación de actividades de salmonicultura que no generan conflictos con las actividades turísticas, aun cuando se identifican algunos puntos focalizados en la comuna de Pucón, donde la sobreposición del área de influencia del centro de cultivo genera conflictos territoriales con el área de influencia turística. En este caso, se presenta tanto el traslape de las actividades como el reconocimiento de la población de que ambas actividades se están desarrollando y compiten generando la percepción de que el desarrollo de una inhabilita o perjudica a la otra, en este caso la acuicultura por sobre la actividad turística.

Esto permite suponer que la percepción de conflicto existente a nivel local, obedece o se ha derivado de los efectos sobre las áreas de influencia indirecta entre las actividades acuícolas y turísticas, dado que ambas hacen usos de los sistemas naturales (recursos hídricos) o que, de acuerdo a lo definido por Riera et al. (2006) y Cruz (2008), obedecen a la creciente preocupación de la población por la calidad de los recursos y la seguridad del lugar donde viven.

Efectivamente, los actores relevantes del territorio en estudio (gubernamental, privado y comunidad organizada) perciben una mayor aptitud territorial para las actividades turísticas, manifestando su preferencia por el desarrollo de estas principalmente porque visualizan, en el turismo, una fuente de empleo favorable para el territorio $y$, aun cuando consideran que la salmonicultura es una fuente de empleo interesante, perciben que el impacto de esta actividad en la empleabilidad es menor, probablemente debido al tamaño y naturaleza de las empresas, las remuneraciones que genera o por el nivel de inversión percibida en la comuna. En general, en todos los sectores consultados se advierte un alto grado de cohesión social en favor del turismo y resistencia para la instalación de nuevos centros de cultivos de salmonídeos, lo cual estaría indicando que los usuarios del territorio están ponderando el uso racional o sustentable del espacio natural (Páez-Osuna, 2001; Primavera, 2006). Esto se evidencia especialmente en la comunidad organizada la cual es el sector que mayor información aporta y la más renuente a la instalación de centros de cultivo o compatibilidad de ambas actividades. Por otro lado, la encuesta permitió identificar un bajo nivel de conocimiento de las normativas ambientales existentes y aplicables a las actividades turísticas y de salmonicultura, lo cual establece una carencia en los actores relevantes, de aspectos fundamentales para la toma de decisiones respecto del uso eficiente del territorio. Esta situación muestra la necesidad de evaluar la percepción de los factores que han de incorporarse a la ordenación del territorio con el propósito de identificar las brechas existentes entre la 
aptitud del medio físico y la percepción de los actores locales (Brugere, 2006; LeónSantana y Hernández, 2008; Sassenberg et al., 2009).

La instalación de centros de cultivos de salmonídeos en los cuerpos fluviales en el área preandina de La Araucanía no ha estado ajena a la opinión de diversas instituciones de Gobierno y privadas que han manifestado públicamente su disconformidad con la presencia de centros de cultivos de especies salmonídeas (OECD/CEPAL, 2005; Wilton, 2009). En este contexto, durante los últimos años, la Secretaría del Convenio sobre la Diversidad Biológica (CDB) y la Organización Mundial para la Agricultura y Alimentación (FAO), han explorado y analizado una nueva forma de abordar la administración de los cultivos acuáticos: el Enfoque Ecosistémico para la Acuicultura (EEA). Este concepto se define como una estrategia para la integración de la actividad dentro de un ecosistema más amplio de tal forma que promueva el desarrollo sustentable, la equidad y la resiliencia de los sistemas ecológicos y sociales que allí se conectan (Soto et al., 2008). Esta estrategia debería responder a tres principios fundamentales que deben garantizar la contribución de la acuicultura al desarrollo sostenible: i) desarrollarse en el contexto de funciones ecosistémicas y de servicios (incluyendo biodiversidad) sin degradar estas más allá de su capacidad de resiliencia; ii) mejorar el bienestar humano y la equidad para todos los grupos de interés relevantes y pertinentes, y iii) la acuicultura debe desarrollarse e integrarse en el contexto de otros sectores relevantes y pertinentes.

Estos principios se ven reflejados en experiencias positivas realizadas en diversos países que podrían extenderse por todo el mundo, en las cuales se promueve el ecoturismo relacionado con la acuicultura. La pesca recreativa en lagos y embalses es muy popular en Australia, España, Europa central, y oriental, especialmente en la Federación de Rusia, Ucrania, Belarús, Moldova y los Estados bálticos (Pérez et al., 2005; Doupé et al., 2005; FAO, 2007; Sassenberg et al., 2009). Otro ejemplo ocurre en Malasia, donde crece el interés por integrar las actividades de acuicultura con el turismo, por ejemplo la piscicultura costera en jaulas y los estanques para pesca de suelta y captura. Asimismo, un ámbito con buenas perspectivas de desarrollo para el ecoturismo acuícola es el de los emplazamientos en alta mar, con la promoción de visitas a emplazamientos de cría en jaulas durante las expediciones a los arrecifes de coral (FAO, 2009).

En el caso particular de la Región de La Araucanía, resulta obligado reflexionar sobre la existencia de un potencial conflicto entre el cada vez mayor desarrollo de la salmonicultura, el turismo y la conservación de los recursos naturales; una cuestión que adquiere especial importancia en el caso de la industria acuícola, dado que la calidad ambiental no es solo un fin en sí mismo sino una condición necesaria para su viabilidad económica (Riera et al., 2006; Soto et al., 2008). De esta forma, la reducción de los problemas de degradación y sobreutilización a los que se ven sometidos los recursos naturales resulta imprescindible para garantizar la supervivencia de la salmonicultura cada vez más globalizada (Díaz y León, 2006; León, 2006).

El desarrollo del hombre y del ambiente es compatible, pero exige planificar el territorio; proceso que debe conducir a armonizar la disponibilidad de los recursos naturales, las condiciones ambientales y las características del territorio, con las actividades económicas y sociales que tienen efecto sobre el entorno natural (Peña-Cortés et al., 2006). Así, la gestión y planificación de los recursos naturales se convierte en un elemento estratégico de la política acuícola del país ya que, la influencia de la salmonicultura sobre el medio ambiente depende no solo de la existencia de impactos ambientales sino también de los esfuerzos públicos y privados dedicados a minimizarlos (FAO, 2009). De esta manera, los antecedentes del medio físico y el análisis de los usuarios del territorio, se constituyen en un aporte significativo para la planificación del territorio (Gómez, 2002; Delgado y Marín, 2005; Geneletti, 2010), ya que representa una visión del potencial conflicto de uso sobre las actividades de salmonicultura y turísticas que demandan, utilizan y comparten servicios ambientales. Esto facilita la formulación de un plan de ordenación y manejo integral del territorio, ya que da cuenta de antecedentes que permiten establecer pautas para asignar, proteger u ordenar los recursos 
y generar procesos de desarrollo local a fin de no desvincular el plan de la problemática real y de las aspiraciones sociales existentes (Molinet et al., 2008; IUCN, 2009).

\section{Conclusiones}

Se confirma el potencial acuícola del área preandina de la Región de La Araucanía por la presencia de áreas con aptitud territorial para la instalación de actividades de salmonicultura que no generan conflictos con las actividades turísticas (11.091 ha con aptitud muy alta y 234.017 ha con aptitud alta). A pesar de esto, se determinó la presencia de cuatro centros de cultivo de salmonídeos como áreas de conflicto con la actividad turística actual, todos ubicados en la comuna de Pucón. Las comunas restantes (Villarrica, Lonquimay, Melipeuco, Curacautín, Vilcún, Curarrehue y Cunco), no presentan conflicto en el uso del territorio para el desarrollo de actividades turísticas o de salmonicultura, debido a que las primeras se realizan en espacios geográficos donde existen restricciones técnicas y legales para las segundas.

Desde el ámbito social, los actores relevantes del territorio (gobierno, privado y comunidad organizada) perciben que el territorio en el cual habitan posee una mayor aptitud para las actividades turísticas que la salmonicultura y manifiestan claramente su preferencia por el desarrollo del turismo por sobre la salmonicultura, principalmente porque visualizan en el primero una fuente de empleo de mayor impacto para el territorio. En contraposición, se observa en la población un bajo nivel de conocimiento de las normativas ambientales existentes y aplicables a las actividades turísticas y de salmonicultura, lo cual permite establecer la carencia de aspectos fundamentales para la toma de decisiones respecto del uso racional o sustentable del territorio.

En síntesis, el desarrollo de la salmonicultura y el turismo en el área preandina de La Araucanía, debe ir acompañado necesaria y oportunamente por el fortalecimiento de la calidad de la gestión de los recursos, por el conocimiento de las capacidades ambientales y sociales realmente disponibles y por la integración de los actores locales en la ordenación, con el objeto de evitar el colapso de la actividad o el surgimiento de conflictos territoriales que desvinculan el plan de la problemática real y de las aspiraciones sociales existentes.

\section{Referencias bibliográficas}

ARMESTO, J. y SMITH-RAMÍREZ, C. Alternativas ecológicas para el manejo del bosque nativo. Ambiente y Desarrollo, 1994, № 11, p. 66-71.

ATLAND, A. y BJERKNES, V. Calidad de agua para el cultivo de smolts en Chile. Santiago de Chile: NIVA Chile, 2009.

BAERISWYL, F. Introducción al ordenamiento territorial rural en Chile. Santiago de Chile: Editorial IICA, Miscellaneous publications series, 2001.

BARREDO, J. Sistemas de Información Geográfica y Evaluación Multicriterio en la ordenación del territorio. Madrid: Editorial RA-MA, 1996.

BARRETT, G.; CANIGGIA, M. \& READ, L. There are more vets than doctors in Chile: social and community impact of globalization of aquaculture in Chile. World Development, 2002, Vol. 30, No 11, p. 1951-2002.

BORJA, Á. Los impactos ambientales de la acuicultura y la sostenibilidad de esta actividad. Boletín del Instituto Español de Oceanografía, 2002, Vol. 18 (1-4), p. 41-49.

BRYCESON, I. Coastal aquaculture developments in Tanzania: sustainable and nonsustainable experiences. Western Indian Ocean Journal of Marine Science, 2002, Vol $1, \mathrm{~N}^{\circ} 1$, p. 1-10.

BRUGERE, C. Can Integrated Coastal Management Solve Agriculture-FisheriesAquaculture Conflicts at the Land-Water Interface? A Perspective from New Institutional Economics. In: HOANH, CH., TUONG, T., GOWING, J. \& HARDY, B. (Editors). Environment and livelihoods in tropical coastal zones: managing agriculture-fishery-aquaculture. Wallingford: CABI, 2006, p. 258-273. 
BRUGERE, C. y RIDLER, N. Perspectivas de la acuicultura mundial en los próximos decenios: análisis de los pronósticos para 2030 de la producción acuícola de los principales países. Roma: FAO Circular de Pesca, $\mathrm{N}^{\circ}$ 1001, 2005.

BUITRAGO, J.; RADA, M.; HERNÁNDEZ, H. \& BUITRAGO, E. A Single-Use Site Selection Technique, Using GIS, for Aquaculture Planning: Choosing Locations for Mangrove Oyster Raft Culture in Margarita Island, Venezuela. Environmental Management, 2005, Vol. 35, № 5, p. 544-556.

CRUZ, H. Conflictos territoriales y movilizaciones ciudadanas: algunas reflexiones sobre las formas de gobernanza territorial actuales. Boletín de la A.G.E., 2008, № 48, p. 375-387.

DÍAZ, S. y LEÓN, J. Actas del Taller Científico: Investigación Ambiental en la Salmonicultura Chilena ¿Gasto o Inversión?. WWF CHILE (Editores). Valdivia: marzo de 2006.

DELGADO L. y MARÍN, V. FES-sistema: un concepto para la incorporación de las sociedades humanas en el análisis medioambiental en Chile. Revista Ambiente y DesarroIlo, 2005, Vol. 21, N³, p. 18-22.

DIRECCIÓN GENERAL DE AGUAS (DGA). Diagnóstico y clasificación de los cursos y cuerpos de agua según objetivos de calidad cuenca del río Imperial. Santiago de Chile: Gobierno de Chile, Ministerio de Obras Públicas, 2004a.

DIRECCIÓN GENERAL DE AGUAS (DGA). Diagnóstico y clasificación de los cursos y cuerpos de agua según objetivos de calidad cuenca del río Toltén. Santiago de Chile: Gobierno de Chile. Ministerio de Obras Públicas, 2004b.

DOUPÉ, R.; SARRE, G.; PARTRIDGE, G.; LYMBERY, A. \& JENKINS, G. What are the prospects for black bream Acanthopagrus butcheri (Munro) aquaculture in salt-affected inland Australia? Aquaculture Research, 2005, Vol. 36, p. 1345-1355.

GENELETTI, D. An approach based on spatial multicriteria analysis to map the na- ture conservation value of agricultural land. Journal of Environmental Management, 2007, Vol. 83, No2, p. 228-235.

GENELETTI, D. Combining stakeholder analysis and spatial multicriteria evaluation to select and rank inert landfill sites. Waste Management, 2010, Vol. 30, № 2, p. 328-337.

GEORGIA STRAIT ALLIANCE. Diverse international group unites in opposition to fish farm expansion. 2002. Disponible en Internet: http://www.georgiastrait.org/?q=node/266

GÓMEZ, D. Ordenación del Territorio. Madrid: Ediciones Mundi-Prensa, 2002.

GOWING, J. W.; TUONG, T. P. \& HOANH, C. Land and water management in coastal zones: Dealing with agriculture, aquaculture, fishery conflicts. In: HOANH, C.; TUONG, T. P.; GOWING, J. W. \& HARDY, B. (Editors). Environment and livelihoods in tropical coastal zones: Managing agriculture, fishery, aquaculture conflicts. Wallingford: CABI, 2006a, p.1-16.

GOWING, J.; TUONG, T.; HOANH, C. \& KHIEM, N. Social and Environmental Impact of Rapid Change in the Coastal Zone of Vietnam: an Assessment of Sustainability Issues. In: HOANH, C.; TUONG, T. P.; GOWING, J. W. \& HARDY, B. (Editors). Environment and livelihoods in tropical coastal zones: Managing agriculture, fishery, aquaculture conflicts. Wallingford: CABI, 2006b, p. 48-60.

HALIDE, H.; STIGEBRANDT, A.; REHBEIN, M. \& MCKINNON, A. Developing a decision support system for sustainable cage aquaculture. Environmental Modelling \& Software, 2009, Vol. 24, p. 694-702.

HOSSAIN, M.; CHOWDHURY, S.; DAS, N.; SHARIFUZZAMAN, S. \& SULTANA, A. Integration of GIS and multicriteria decision analysis for urban aquaculture development in Bangladesh. Landscape and Urban Planning, 2009, Vol. 90, N³-4, p. 119-133.

INTERNATIONAL UNION FOR CONSERVATION OF NATURE (IUCN). Guide for the Sustainable Development of Mediterranean Aquaculture. Interaction between Aquaculture and the Environment. Gland/Malaga: IUCN, 2007. 
INTERNATIONAL UNION FOR CONSERVATION OF NATURE (IUCN). Guide for the Sustainable Development of Mediterranean Aquaculture 2. Aquaculture site selection and site management. Gland/Malaga: IUCN, 2007.

ISRAEL, D. Assessing the severity of problems of aquaculture in Laguna de Bay: practitioners' perspectives. Philippine Institute for Development Studies. Policy Notes, № 2007 04, 2007. Disponible en internet: http://dirp4. pids.gov.ph/ris/pn/pidspn0704.pdf

JAYANTHI, M.; REKHA, P.; KAVITHA, N. \& RAVICHANDRAN, P. Assessment of impact of aquaculture on Kolleru Lake (India) using remote sensing and Geographical Information System. Aquaculture Research, 2006, Vol. 37, p. 1617-1626.

LABORATORIO DE PLANIFICACIÓN TERRITORIAL/UNIVERSIDAD CATÓLICA DE TEMUCO (LPT-UCT). Catastro oferta turística regional. SERNATUR, Región de La Araucanía. Temuco, 2002.

LABORATORIO DE PLANIFICACIÓN TERRITORIAL/UNIVERSIDAD CATÓLICA DE TEMUCO (LPT-UCT). Plan Regional de Desarrollo Urbano y Territorial. IX Región de La Araucanía. Chile. Temuco: MINVU, 2005.

LEÓN, J. Informe Técnico de Consultoría Sinopsis de los Impactos y la Gestión Ambiental de la Salmonicutura Chilena. Puerto Montt: WWF, 2006.

LEÓN-SANTANA, M. \& HERNÁNDEZ, J. Analysis: Optimum management and environmental protection in the aquaculture industry. Ecological Economics, 2008, Vol. 64, p. 849-857.

MACÍAS, J.; DEL CASTILLO, F.; VILLARÍA, R. y GAITEIRO, J. Zonas de interés para cultivos marinos: identificación y gestión para el desarrollo ordenado de la acuicultura en Andalucía. Boletín del Instituto Español de Oceanografía, 2005, Vol. 21, № (1-4), p. 47-55.

MARDONES, M.; JARA, J. Y VARGAS, J. El patrón hidrográfico de la cuenca del río Blanco: control tectónico y geomorfológico. Revista de Geografía Norte Grande, 2007, № 38, p. 79-98.
MOLINET, C.; ARÉVALO, A.; DÍAZ, M. y DÍAZ, P. Uso del borde costero en el mar interior de la Región de Aysén y de Los Lagos: escalas e interacción de los procesos de pesca y acuicultura. En: LOVATELLI, A.; FARÍAS, A. y URIARTE, I. (Editores). Estado actual del cultivo y manejo de moluscos bivalvos y su proyección futura: factores que afectan su sustentabilidad en América Latina. Roma: FAO Actas de Pesca y Acuicultura, 2008, Nº 12 , p. 237-247.

NOAKES, D.; FANG, L.; HIPEL, K. \& KILGOUR, D. An examination of the salmon aquaculture conflict in British Columbia using the graph model for conflict resolution. Fisheries Management and Ecology, 2003, No 10, p. 123-137.

ORGANIZACIÓN MUNDIAL DEL TURISMO (OMT). Tendencias de los Mercados Turísticos. Edición 2006 - Las Américas. Madrid: OMT, 2007.

ORGANISATION FOR ECONOMIC COOPERATION AND DEVELOPMENT (OECD)/ COMISIÓN ECONÓMICA PARA AMÉRICA LATINA (CEPAL). Evaluaciones del desempeño ambiental Chile. Santiago de Chile: OECD/CEPAL, 2005. Disponible en Internet: http://www.ine.cl/canales/menu/OCDE/ OCDE_Informes/InformeMedioAmbiente2005texto.pdf

ORGANIZACIÓN DE LAS NACIONES UNIDAS PARA LA AGRICULTURA Y LA ALIMENTACIÓN (FAO). El Estado Mundial de la Pesca y la Acuicultura 2006. Roma: Departamento de Pesca y Acuicultura de la FAO, 2007.

ORGANIZACIÓN DE LAS NACIONES UNIDAS PARA LA AGRICULTURA Y LA ALIMENTACIÓN (FAO). El Estado Mundial de la Pesca y la Acuicultura 2008. Roma: Departamento de Pesca y Acuicultura de la FAO, 2009.

PÁEZ-OSUNA, F. The Environmental Impact of Shrimp Aquaculture: Causes, Effects, and Mitigating Alternatives. Environmental Management, 2001, Vol. 28, No 1, p. 131140. 
PEÑA-CORTÉS, F. y MARDONES, M. Planificación ecológica del curso inferior del río Itata, VIII Región Chile. Revista Geográfica de Chile Terra Australis, 1999, № 44, p. 45-62.

PEÑA-CORTÉS, F., GUTIÉRREZ P., REBOLLEDO, G., ESCALONA M., HAUENSTEIN, E., BERTRÁN, C., SCHLATTER, R. y TAPIA, J. Determinación del nivel de antropización de humedales como criterio para la planificación ecológica de la cuenca del lago Budi, IX Región de La Araucanía, Chile. Revista de Geografía Norte Grande. 2006, №.36, p. 75-91.

PEÑA-CORTÉS, F.; ESCALONA-ULLOA, M.; REBOLLEDO, G.; PINCHEIRA-ULBRICH, J. y TORRES ÁlVAREZ, O. Efecto del cambio en el uso del suelo en la economía local: Una perspectiva histórica en el borde costero de La Araucanía, Sur de Chile. En: CONFALONIERI, U.; MENDOZA, M. \& FERNÁNDEZ, L. (Editores). Efecto de los cambios globales sobre la salud humana y la seguridad alimentaria. Buenos Aires: CYTED, 2009, p. 184-197.

PÉREZ, E.; MUIR, J. \& ROSS, L. Coastal aquaculture and sustainable livelihoods in Mecoacan, Tabasco, Mexico. Universidad y Ciencia, 2002, Vol. 18, № 35.

PÉREZ, O.; TELFER, T. \& ROSS, L. Geographical information systems-based models for offshore floating marine fish cage aquaculture site selection in Tenerife, Canary Islands. Aquaculture Research, 2005, Vol. 36, p.946961.

PICHARA, C.; MARDONES, A.; SALAS, R.; REYNE, O.; VEGA, R.; VALDEBENITO, I.; ENCINA, F.; ÁlVAREZ, F. y ZAMORANO, J. Prospectiva Tecnológica de las Pisciculturas de la Región de La Araucanía. Temuco: Proyecto INNOVA-CORFO, Nodo Tecnológico Acuícola, Enlace Acuícola. Escuela de Acuicultura de la Universidad Católica de Temuco, 2007.

PRIMAVERA, J. Overcoming the impacts of aquaculture on the coastal zone. Ocean \& Coastal Management, 2006, Vol. 49, p. 531545.
QUIROZ, J. y CONSULTORES ASOCIADOS. Informe Económico Salmonicultura 2006. Santiago de Chile: Instituto Tecnológico del Salmón (INTESAL), Asociación de la Industria del Salmón de Chile A.G, 2006.

RADIARTA, N.; SAITOH, S. \& MIYAZONO, A. GIS-based multi-criteria evaluation models for identifying suitable sites for Japanese scallop (Mizuhopecten yessoensis) aquaculture in Funka Bay, southwestern Hokkaido, Japan. Aquaculture, 2008, Vol. 284, № 1-4, p. 127-135.

RIERA, A.; CAPÓ, J. y PALMER, T. ¿Existe un conflicto entre la globalización del turismo y los recursos naturales? Economía Agraria y Recursos Naturales, 2006, Vol. 6, $\mathrm{N}^{\circ} 12$, p. 169-186.

RIVERA H. Aplicación de la evaluación multicriterio para la asignación de funciones al territorio de la Reserva Nacional Valdivia Corporación Nacional Forestal. Valdivia: Departamento de Manejo y Desarrollo Forestal. Proyecto Manejo Sustentable del Bosque Nativo. Subproyecto Plan de Ordenación Reserva Nacional Valdivia (CONAF / GTZ), 2001.

SASSENBERG, U.; HULL, J. \& JODICE, L. Exploring the impact of innovation in promoting sustainable tourism development: The role of key stakeholders on the Top of the South Aquaculture and Seafood Trail in Nelson/ Marlborough, New Zealand. Auckland: Proceedings of the Environmental Research Event 2009, Noosa, QLD. Disponible en Internet: http://espace.library.uq.edu.au/eserv/ UQ:179622/Sassenberg_-_ERE2009.pdf

SERVICIO NACIONAL DE PESCA (SERNAPESCA). Anuario estadístico de Pesca. 2009. Disponible en Internet: http://www.sernapesca.cl/index.php?option=com_remository\&lte $\mathrm{mid}=54 \&$ func $=$ select\&id $=382$

SERVICIO NACIONAL DE TURISMO (SERNATUR). Cartografía áreas turísticas prioritarias en Chile. Santiago de Chile: Departamento de Planificación SERNATUR, 2007. 
SERVICIO NACIONAL DE TURISMO (SERNATUR). Turismo Informe anual 2008. Santiago de Chile: Departamento de Planificación SERNATUR, 2009.

SIGTUR. Informe Proyecto: Aplicación cartografía digital cobertura oferta turística provincias de Malleco y Cautín. Temuco: 2002.

SOTO, D.; AGUILAR-MANJARREZ, J. \& HISHAMUNDA, N. (Editores). Building an ecosystem approach to aquaculture. Roma: FAO Fisheries and Aquaculture Proceedings, $N^{\circ} 14,2008$.

SURYANATA, K. \& UMEMOTO, K. Beyond environmental impact: articulating the "intangibles" in a resource conflict. Geoforum, 2005, Vol. 36, N6, p. 750-760.

STRAHLER, A. Quantitative analysis of watershed geomorphology. American Geophysical Union Transactions, 1957, Vol. $38, N^{\circ} 6$, p. $913-20$

STRAHLER, A. N. Quantitative geomorphology of drainage basins and channel networks. Handbook of Applied Hydrology, 1964, Section 4 - I, p. 39-76.

SUBSECRETARIA DE PESCA (SUBPESCA) Informe Sectorial de Pesca y Acuicultura 2009. Santiago de Chile: Departamento de Análisis Sectorial. Disponible en Internet: http://www.subpesca.cl/mostrararchivo. asp?id=9750

UNION OF BRITISH COLUMBIAN INDIAN CHIEFS. Fish farms: Zero tolerance. Indian salmon don't do drugs. 2004. Disponible en Internet: http://www.ubcic.bc.ca/Resources/fishfarmpaper.htm

WILTON, G. Acuicultura está perjudicando desarrollo turístico de Aysén. Asociación de periodistas de turismo de Chile. 2009. Disponible en Internet:

h t t p : / / www.apturchile.cl/index. php? Itemid=13\&id=54\&option=com _ content\&task=view

WORLD TRAVEL AND TOURISM COUNCIL (WTTC) Travel \& Tourism economic impact. Latin America, 2010. Disponible en Internet:

http://www.wttc.org/bin/pdf/temp/latinamerica.html

ZUÑIGA, S.; ACUÑA, E. y BODINI, A. Un modelo para la localización óptima de cultivos acuicolas en estanques: El caso de la Región de Atacama - Chile. Revista Visión de Futuro, 2006, Vol. 6, № 2, p. 185-195. 
